\title{
Loss of Vision
}

National Cancer Institute

\section{Source}

National Cancer Institute. Loss of Vision. NCI Thesaurus. Code C50638.

A condition in which the ability to see is impaired. 